\title{
Global optimal solutions for noncyclic mappings in $G$-metric spaces
}

Saeed Shabani and Abdolrahman Razani 


\title{
GLOBAL OPTIMAL SOLUTIONS FOR NONCYCLIC MAPPINGS IN $G$-METRIC SPACES
}

\author{
SAEED SHABANI AND ABDOLRAHMAN RAZANI
}

Received 5 June, 2012

\begin{abstract}
In this paper, the existence of solutions of some minimization problems for noncyclic mappings in $G$-metric spaces is studied. Our results can be considered as an extension of Abkar and Gabeleh's result [Global Optimal Solutions of Noncyclic Mappings in Metric Spaces, J. Optim. Theory. Appl. 153 (2011), 298-305] to the case of $G$-metric spaces.
\end{abstract}

2000 Mathematics Subject Classification: 41A65; 46B20; 47H10

Keywords: $G$-metric space, noncyclic mapping, minimization problem

\section{INTRODUCTION}

In 2011, Abkar et al. [2] studied the existence of solutions of some specific minimization problems for noncyclic mappings in metric spaces. In 2006, Mustafa et al. [11] introduced the $G$-metric spaces as a generalization of the notion of metric spaces. Fixed point results and other results in $G$-metric spaces have been proved by a number of authors, see, e.g., $[1,3-5,12,14,15]$. In this paper we investigate some minimization problems for noncyclic mappings in $G-$ metric spaces. This work extends results of Abkar et al. [2] to the case of $G$-metric spaces.

\section{Preliminaries}

Throughout this paper, $N$ is the set of all natural numbers and $R$ is the set of all real numbers. Generalizations of the notion of a metric space have been proposed by Gabler [8,9] and by Dhage [6,7]. Mustafa et al. [11] introduced a more appropriate notion of a generalized metric space as following.

Definition 1. Let $X$ be a nonempty set, and $G: X \times X \times X \rightarrow R^{+}$be a function satisfying the following conditions:

(1) $G(x, y, z)=0$ if $x=y=z$,

(2) $0<G(x, x, y)$ for all $x, y \in X$ with $x \neq y$,

The second author was supported in part by Imam Khomeini International University, Grant No.751168-91. 
(3) $G(x, x, y) \leq G(x, y, z)$ for all $x, y, z \in X$ with $y \neq z$,

(4) $G(x, y, z)=G(x, z, y)=G(y, z, x)=\cdots$,

(5) $G(x, y, z) \leq G(x, w, w)+G(w, y, z)$ for all $x, y, z, w \in X$,

The function $G$ is called a generalized metric, or, a $G-$ metric on $X$, and the pair $(X, G)$ is called a $G-$ metric space.

Example 1. ([11, Example 6.3]) Let $(X, d)$ be a metric space and define the functions $G_{s}$ and $G_{m}$ with

$$
\begin{gathered}
G_{s}(x, y, z)=d(x, y)+d(y, z)+d(x, z), \quad \forall x, y, z \in X \\
G_{m}(x, y, z)=\max \{d(x, y), d(y, z), d(x, z)\}, \quad \forall x, y, z \in X
\end{gathered}
$$

Then $\left(X, G_{s}\right)$ and $\left(X, G_{m}\right)$ are $G-$ metric space.

Now, we recall some of the basic concepts for $G$-metric spaces from ([11]).

Definition 2. Let $(X, G)$ be a $G-$ metric space, and $\left\{x_{n}\right\}$ be a sequence of points of $X$, we say that $\left\{x_{n}\right\}$ is $G$-convergent to $\mathrm{x}$ and write $x_{n} \longrightarrow x$ if $\lim _{n, m \rightarrow \infty} G(x$, $\left.x_{n}, x_{m}\right)=0$, that is, for any $\epsilon>0$, there exists $n_{0} \in N$ such that $G\left(x, x_{n}, x_{m}\right)<\epsilon$, for all $n, m \geq n_{0}$.

Proposition 1. Let $(X, G)$ be a $G$-metric space, then the following are equivalent.

(1) $\left\{x_{n}\right\}$ is $G$-convergent to $x$.

(2) $\lim _{n \rightarrow \infty} G\left(x, x_{n}, x_{n}\right)=0$.

(3) $\lim _{n \rightarrow \infty} G\left(x, x, x_{n}\right)=0$.

Definition 3. Let $(X, G)$ be a $G$-metric space, a $\left\{x_{n}\right\}$ is called $G$-Cauchy for any $\epsilon>0$, there exists $n_{0} \in N$ such that $G\left(x_{n}, x_{m}, x_{l}\right)<\epsilon$, for all $n, m, l \geq n_{0}$ that is $\lim _{n, m, l \rightarrow \infty} G\left(x_{n}, x_{m}, x_{l}\right)=0$

Proposition 2. Let $(X, G)$ be a $G$-metric space, then the following are equivalent.

(1) $\left\{x_{n}\right\}$ is $G-$ Cauchy.

(2) For any $\epsilon>0$, there exists $n_{0} \in N$ such that $G\left(x_{n}, x_{m}, x_{m}\right)<\epsilon$, for all $n, m \geq$ $n_{0}$

Definition 4. Let $\left(X_{1}, G_{1}\right)$ and $\left(X_{2}, G_{2}\right)$ be $G$-metric spaces. A function $f$ : $\left(X_{1}, G_{1}\right) \rightarrow\left(X_{2}, G_{2}\right)$ is $G-$ continuous at a point $a \in X$ if for any $\epsilon>0$, there exists $\delta>0$ such that $x, y \in X_{1}, G_{1}(a, x, y)<\delta$ implies $G_{2}(f(a), f(x), f(y))<\epsilon$. A function $\mathrm{f}$ is $G$-continuous on $X$ if and only if it is $G$-continuous at all $a \in X$.

Proposition 3. Let $\left(X_{1}, G_{1}\right)$ and $\left(X_{2}, G_{2}\right)$ be $G$-metric spaces. A function $f$ : $\left(X_{1}, G_{1}\right) \rightarrow\left(X_{2}, G_{2}\right)$ is $G$-continuous at a point $x \in X$ if and only if whenever $\left\{x_{n}\right\}$ is $G$-convergent to $X,\left\{f\left(x_{n}\right)\right\}$ is $G$-convergent to $f(x)$.

Definition 5. A $G$-metric space $(X, G)$ is said to be $G$-complete if every $G$ Cauchy sequence in $(X, G)$ is $G$-convergent in $(X, G)$. 
Definition 6. Let $(X, G)$ be a $G-$ metric space. A $G-$ Ball with center $x_{0}$ and radius $r$ is

$$
B_{G}\left(x_{0}, r\right)=\left\{x \in X: G\left(x_{0}, y, y\right)<r\right\} .
$$

Definition 7. Let $(X, G)$ be a $G$-metric space and $\epsilon>0$ be given, then a set $A \subset X$ is called $\epsilon-$ net of $(X, G)$ if given any $x$ there is at last one point $a \in A$ such that $x \in B_{G}(a, \epsilon)$. If the $A$ is finite then $A$ is called a finite $\epsilon-$ net of $(X, G)$. Note that if $A$ is an $\epsilon-$ net then $X=\bigcup_{a \in A} B_{G}(a, \epsilon)$.

Definition 8. A $G$-metric space $(X, G)$ is called $G$-totally bounded if for every $\epsilon>0$ there exists a finite $\epsilon-$ net.

Definition 9. A $G$-metric space $(X, G)$ is called $G$-compact space if it is $G$-complete and $G$-totally bounded.

Proposition 4. Let $(X, G)$ be a $G$-metric space, then the following are equivalent.

(1) $(X, G)$ is a $G$-compact space.

(2) $(X, G)$ is $G$-sequentially compact, that is, if the sequence $\left\{x_{n}\right\} \subset X$ is such that $\sup \left\{G\left(x_{n}, x_{m}, x_{l}\right): n, m, l \in N\right\}<\infty$, then $\left\{x_{n}\right\}$ has a $G$-convergent subsequence.

Theorem 1 ([12], Theorem 2.1). Let $(X, G)$ be a $G$-metric space and $T: X \rightarrow X$ be a mapping which satisfies the following condition, for all $x, y, z \in X$,

$$
\begin{gathered}
G(T(x), T(y), T(z)) \leq \quad k \max \{G(x, y, z), G(x, T(x), T(x)), G(y, T(y), T(y)), \\
G(z, T(z), T(z)), G(x, T(y), T(y)), \\
G(y, T(z), T(z)), G(z, T(x), T(x))\},
\end{gathered}
$$

where $k \in[0,1 / 2)$. Then $T$ has a unique fixed point (say $u$ ) and $T$ is $G$-continuous at $u$.

Definition 10. Let $A, B, C$ be subsets of a $G$-metric space $(X, G)$. A mapping $T: A \cup B \cup C \rightarrow A \cup B \cup C$ is called relatively $G$-nonexpansive if

$$
G(T(x), T(y), T(z)) \leq G(x, y, z), \quad \forall(x, y, z) \in A \times B \times C .
$$

Definition 11. Let $(X, G)$ be a $G-$ metric space and $A, B, C \subset X$, then

$$
\operatorname{dist}(A, B, C)=\inf \{G(a, b, c): a \in A, b \in B, c \in C\} .
$$

Example 2. Let $R$ be equipped with the usual metric, and $A=[-1,0]$ and $B=$ $N_{o}$ and $C=N_{e}$ where $N_{o}$ and $N_{e}$ are the set of odd natural numbers and even natural numbers, respectively. Let $G_{m}(x, y, z)=\max \{|x-y|,|x-z|,|y-z|\}$, then $\operatorname{dist}(A, B, C)=2$.

Definition 12. Let $(X, G)$ be a $G$-metric space and $A, B, C \subset X, T: A \cup B \cup$ $C \rightarrow A \cup B \cup C$ is said noncyclic mapping, if

$$
T(A) \subset A, \quad T(B) \subset B, \quad T(C) \subset C .
$$


We consider the following minimization problem: Find

$$
\begin{array}{ll}
\min _{a \in A}\{G(a, T(a), T(a))\}, & \min _{b \in B}\{G(b, T(b), T(b))\}, \\
\min _{b \in B}\{G(c, T(c), T(c))\}, & \min _{(a, b, c) \in A \times B \times C}\{G(a, b, c)\}
\end{array}
$$

We say that $\left(x^{\star}, y^{\star}, z^{\star}\right) \in A \times B \times C$ is a solution of above problem, if

$$
T x^{\star}=x^{\star}, \quad T y^{\star}=y^{\star}, T z^{\star}=z^{\star},
$$

and

$$
G\left(x^{\star}, y^{\star}, z^{\star}\right)=\operatorname{dist}(A, B, C) .
$$

Definition 13. Let $(X, G)$ be a $G-$ metric space and $A, B, C \subset X$, we set

$$
\begin{aligned}
& A_{0}=\{a \in A: G(a, b, c)=\operatorname{dist}(A, B, C), \text { for some } b \in B, c \in C\} \\
& B_{0}=\{b \in B: G(a, b, c)=\operatorname{dist}(A, B, C), \text { for some } a \in A, c \in C\} \\
& C_{0}=\{c \in C: G(a, b, c)=\operatorname{dist}(A, B, C), \text { for some } a \in A, b \in B\}
\end{aligned}
$$

Definition 14. Let $(X, G)$ be a $G-$ metric space and $A, B, C$ be nonempty subsets of $X$, with $A_{0} \neq \varnothing$. We say that $A, B, C$ have $P$-property iff

$$
\left\{\begin{array}{l}
G\left(x_{1}, y_{1}, z_{1}\right)=\operatorname{dist}(A, B, C) \\
G\left(x_{2}, y_{2}, z_{2}\right)=\operatorname{dist}(A, B, C) \\
G\left(x_{3}, y_{3}, z_{3}\right)=\operatorname{dist}(A, B, C)
\end{array}\right.
$$

then

$$
G\left(x_{1}, x_{2}, x_{3}\right)=G\left(y_{1}, y_{2}, y_{3}\right)=G\left(z_{1}, z_{2}, z_{3}\right),
$$

where $x_{1}, x_{2}, x_{3} \in A_{0}$ and $y_{1}, y_{2}, y_{3} \in B_{0}$ and $z_{1}, z_{2}, z_{3} \in C_{0}$.

The above definition were found in the case of metric space in ([13]).

Example 3. Let $A, B, C$ be nonempty subsets of a $G$-metric space $(X, G)$ such that $A_{0} \neq \varnothing$ and $\operatorname{dist}(A, B, C)=0$, then $A, B, C$ have $P$-property.

Definition 15. Let $(X, G)$ be a $G-$ metric space and $T: X \rightarrow X$ be a mapping. $T$ is called expansive if for all $x, y, z \in X$,

$$
G(T(x), T(y), T(z)) \geq G(x, y, z) .
$$

Definition 16. Let $(X, G)$ be a $G-$ metric space and $T: X \rightarrow X$ be a mapping. $T$ is said to be asymptotically regular iff $\lim _{n \rightarrow \infty} G\left(T^{n} x, T^{n+1} x, T^{n+1} x\right)=0$, for all $x \in X$. 


\section{MAIN RESULTS}

We start this section with the following theorem.

Theorem 2. Let $A, B, C$ be nonempty and closed subsets of a $G$-complete space $(X, G)$ such that $A_{0} \neq \varnothing$ and $A, B, C$ satisfies the $P$-property. Let $T: A \cup B \cup C \rightarrow$ $A \cup B \cup C$ be a noncyclic mapping. Suppose that

(1) $\left.T\right|_{A}$ be a mapping which satisfies in (2.1).

(2) $T$ is relatively $G-$ nonexpansive.

Then the minimization problem (2.2) has a solution.

Proof. If $x \in A_{0}$, then there exist $y \in B$ and $z \in C$ such that $G(x, y, z)$ $=\operatorname{dist}(A, B, C)$. Since $T$ is relatively $G-$ nonexpansive then

$$
G(T(x), T(y), T(z)) \leq G(x, y, z)=\operatorname{dist}(A, B, C)
$$

Hence $T x \in A_{0}$.

Let $x_{0} \in A_{0}$ by Theorem 1 if $x_{n}=T^{n}\left(x_{0}\right)$ then $x_{n} \stackrel{G}{\longrightarrow} x^{\star}$ where $x^{\star}$ is unique fixed point of $T$ in $A$. Since $x_{0} \in A_{0}$ there exist $y_{0} \in B$ and $z_{0} \in C$ such that $G\left(x_{0}, y_{0}, z_{0}\right)=\operatorname{dist}(A, B, C)$. Since $x_{1}=T x_{0} \in A_{0}$, there exist $y_{1} \in B$ and $z_{1} \in C$ such that $G\left(x_{1}, y_{1}, z_{1}\right)=\operatorname{dist}(A, B, C)$. Using this process, we have a sequence $\left\{y_{n}\right\}$ in $B$ and $\left\{z_{n}\right\}$ in $C$ such that

$$
G\left(x_{n}, y_{n}, z_{n}\right)=\operatorname{dist}(A, B, C) \quad \forall n \in N \cup\{0\} .
$$

Since $A, B, C$ have the $P$-property, we have for all $m, n, l \in N \cup\{0\}$

$$
G\left(x_{n}, x_{m}, x_{l}\right)=G\left(y_{n}, y_{m}, y_{l}\right)=G\left(z_{n}, z_{m}, z_{l}\right) .
$$

This implies that $\left\{y_{n}\right\}$ and $\left\{z_{n}\right\}$ are $G-$ Cauchy sequences, and there exist $y^{\star} \in B$ and $z^{\star} \in C$ such that $y_{n} \stackrel{G}{\longrightarrow} y^{\star}$ and $z_{n} \stackrel{G}{\longrightarrow} z^{\star}$ Thus

$$
G\left(x^{\star}, y^{\star}, z^{\star}\right)=\lim _{n \rightarrow \infty} G\left(x_{n}, y_{n}, z_{n}\right)=\operatorname{dist}(A, B, C)
$$

Since

$$
G\left(T\left(x^{\star}\right), T\left(y^{\star}\right), T\left(z^{\star}\right)\right) \leq G\left(x^{\star}, y^{\star}, z^{\star}\right)=\operatorname{dist}(A, B, C)
$$

Therefore by the $P$-property, we have

$$
G\left(x^{\star}, T\left(x^{\star}\right), T\left(x^{\star}\right)\right)=G\left(y^{\star}, T\left(y^{\star}\right), T\left(y^{\star}\right)\right)=G\left(z^{\star}, T\left(z^{\star}\right), T\left(z^{\star}\right)\right)
$$

Thus $\left(x^{\star}, y^{\star}, z^{\star}\right) \in A \cup B \cup C$ is a solution of the minimization problem (2.2).

Example 4. Let $R$ be equipped with the usual metric, and $G_{m}(x, y, z)=\max \{\mid x-$ $y|| x-,z|| y-z \mid$,$\} . Let A=[-2,0]$ and $B=\{1\}$ and $C=[2,3]$. It is obvious that 
$A_{0}=\{0\}, B_{0}=\{1\}, C_{0}=\{2\}$. Define $T: A \cup B \cup C \rightarrow A \cup B \cup C$ with

$$
T(x)= \begin{cases}\frac{x}{4} & x \in A \\ 1 & x \in B \\ \frac{x+2}{2} & x \in C\end{cases}
$$

It is easy to check that all the conditions of Theorem 2 hold. Therefore, the minimization problem (2.2) has a solution $\left(x^{\star}, y^{\star}, z^{\star}\right)=(0,1,2)$.

Theorem 3. Let $A, B, C$ be nonempty subsets of a $G$-complete space $(X, G)$ such that $A$ is $G$-compact and $B$ and $C$ are $G$-closed. Let $A_{0} \neq \varnothing$ and $A, B, C$ satisfy the $P$-property. Let $T: A \cup B \cup C \rightarrow A \cup B \cup C$ be a noncyclic mapping. Then the minimization problem (2.2) has a solution provided that the following conditions are satisfied:

(1) $T$ is relatively $G-$ nonexpansive.

(2) $\left.T\right|_{A}$ is a $G$-expansive.

(3) $\left.T\right|_{B}$ and $\left.T\right|_{C}$ be mappings which satisfy in (2.1).

Proof. If $x \in A_{0}$, and $x_{n+1}=T x_{n},(n \in N \cup\{0\})$. By argument similar in the proof of Theorem 2 we obtain that $T\left(A_{0}\right) \subset A_{0}$ and there exist $y_{n}$ in $B$ and $z_{n}$ in $C$ such that

$$
G\left(x_{n}, y_{n}, z_{n}\right)=\operatorname{dist}(A, B, C) \quad \forall n \in N \cup\{0\} .
$$

Since $A$ is $G$-compact, by Proposition 4 there exist a subsequence $\left\{x_{n_{k}}\right\}$ of the $\left\{x_{n}\right\}$ such that $x_{n_{k}} \stackrel{G}{\longrightarrow} x^{\star} \in A$. Since $A, B, C$ satisfy the $P$-property,

$$
G\left(x_{n_{k}}, x_{n_{s}}, x_{n_{l}}\right)=G\left(y_{n_{k}}, y_{n_{s}}, y_{n_{l}}\right)=G\left(z_{n_{k}}, z_{n_{s}}, z_{n_{l}}\right), \quad(k, s, l \in N) .
$$

This implies that $\left\{y_{n}\right\}$ and $\left\{z_{n}\right\}$ are $G$-Cauchy sequences and there exist $y^{\star} \in B$ and $z^{\star} \in C$ such that $y_{n_{k}} \stackrel{G}{\longrightarrow} y^{\star}$ and $z_{n_{k}} \stackrel{G}{\longrightarrow} z^{\star}$. Thus

$$
G\left(x^{\star}, y^{\star}, z^{\star}\right)=\lim _{n \rightarrow \infty} G\left(x_{n_{k}}, y_{n_{k}}, z_{n_{k}}\right)=\operatorname{dist}(A, B, C)
$$

Now we prove that $x^{\star}, y^{\star}, z^{\star} \in F(T)$. Since $T$ is relatively $G$-nonexpansive,

$$
G\left(T^{2}\left(x^{\star}\right), T^{2}\left(y^{\star}\right), T^{2}\left(z^{\star}\right)\right)=G\left(T\left(x^{\star}\right), T\left(y^{\star}\right), T\left(z^{\star}\right)\right)=\operatorname{dist}(A, B, C) .
$$

Since $A, B, C$ satisfy the $P$-property, we have

$$
G\left(x^{\star}, T\left(x^{\star}\right), T\left(x^{\star}\right)\right)=G\left(y^{\star}, T\left(y^{\star}\right), T\left(y^{\star}\right)\right)=G\left(z^{\star}, T\left(z^{\star}\right), T\left(z^{\star}\right)\right),
$$

and

$$
\begin{aligned}
G\left(T\left(x^{\star}\right), T^{2}\left(x^{\star}\right), T^{2}\left(x^{\star}\right)\right) & =G\left(T\left(y^{\star}\right), T^{2}\left(y^{\star}\right), T^{2}\left(y^{\star}\right)\right) \\
& =G\left(T\left(z^{\star}\right), T^{2}\left(z^{\star}\right), T^{2}\left(z^{\star}\right)\right) .
\end{aligned}
$$

Now let $T y^{\star} \neq T^{2} y^{\star}$, since $\left.T\right|_{B}$ satisfies in (2.1),

$$
G\left(T\left(y^{\star}\right), T\left(T\left(y^{\star}\right)\right), T\left(T\left(y^{\star}\right)\right)\right) \leq k G\left(y^{\star}, T\left(y^{\star}\right), T\left(y^{\star}\right)\right)
$$


Thus since $\left.T\right|_{A}$ is a $G$-expansive, we have

$$
\begin{aligned}
G\left(T\left(y^{\star}\right), T^{2}\left(y^{\star}\right), T^{2}\left(y^{\star}\right)\right) & =G\left(T\left(y^{\star}\right), T\left(T\left(y^{\star}\right)\right), T\left(T\left(y^{\star}\right)\right)\right) \\
\leq & k G\left(y^{\star}, T\left(y^{\star}\right), T\left(y^{\star}\right)\right) \\
& =k G\left(x^{\star}, T\left(x^{\star}\right), T\left(x^{\star}\right)\right) \\
\leq & k G\left(T\left(x^{\star}\right), T^{2}\left(x^{\star}\right), T^{2}\left(x^{\star}\right)\right) \\
& =k G\left(T\left(y^{\star}\right), T^{2}\left(y^{\star}\right), T^{2}\left(y^{\star}\right)\right),
\end{aligned}
$$

which is a contraction. Therefore $T y^{\star}=T^{2} y^{\star}$. A similar argument implies that $T z^{\star}=T^{2} z^{\star}$. Thus $x^{\star}=T\left(x^{\star}\right)$ and $y^{\star}=T\left(y^{\star}\right)$ and $z^{\star}=T\left(z^{\star}\right)$.

Example 5. Let $X=R^{3}$ and

$$
\begin{aligned}
G\left(\left(x_{1}, y_{1}, z_{1}\right),\left(x_{2}, y_{2}, z_{2}\right),\left(x_{3}, y_{3}, z_{3}\right)\right)= & \max \left\{G_{m}\left(x_{1}, x_{2}, x_{3}\right),\right. \\
& \left.G_{m}\left(y_{1}, y_{2}, y_{3}\right), G_{m}\left(z_{1}, z_{2}, z_{3}\right)\right\},
\end{aligned}
$$

where $G_{m}(x, y, z)=\max \{|x-y|,|x-z|,|y-z|\}$. Let $A=\{(x, 0,0):-1 \leq x \leq$ $0\}$ and $B=\{(0, y, 0): 0 \leq x \leq 1\}$ and $C=\{(0,0, z):-1 \leq z \leq 1\}$. It is obvious that $A_{0}=B_{0}=C_{0}=\{(0,0,0)\}$ and $\operatorname{dist}(A, B, C)=0$, therefore $A, B, C$ have the $P$-property. Define $T: A \cup B \cup C \rightarrow A \cup B \cup C$ with

$$
T(x, 0,0)=(-x, 0,0), T(0, y, 0)=\left(0, \frac{y}{4}, 0\right) \text { and } T(0,0, z)=\left(0,0, \frac{z}{4}\right) .
$$

It is easy to check that all the conditions of Theorm 3 hold. Therefore the minimization problem (2.2) has a solution $x^{\star}=y^{\star}=z^{\star}=(0,0,0)$.

Theorem 4. Let $A, B, C$ be nonempty subsets of a $G$-complete space $(X, G)$ such that $A$ is $G$-compact and $B$ and $C$ are $G$-closed.Let $A_{0} \neq \varnothing$ and $A, B, C$ satisfy the $P$-property. Let $T: A \cup B \cup C \rightarrow A \cup B \cup C$ be a noncyclic mapping. Then the minimization problem (2.2) has a solution provided that the following conditions are satisfied:

(1) $T$ is relatively $G-$ nonexpansive.

(2) $\left.T\right|_{A}$ is $G$-continuous and asymptotically regular.

Proof. Let $\left\{x_{n}\right\},\left\{y_{n}\right\},\left\{z_{n}\right\},\left\{x_{n_{k}}\right\},\left\{y_{n_{k}}\right\},\left\{z_{n_{k}}\right\}, x_{G}^{\star}, y^{\star}$ and $z^{\star}$ be as in Theorem 3. We have $x_{n_{k}} \longrightarrow x^{\star} \in A, y_{n_{k}} \stackrel{G}{\longrightarrow} y^{\star} \in B, z_{n_{k}} \longrightarrow z^{\star} \in C$ and $G\left(x^{\star}, y^{\star}, z^{\star}\right)=$ $\operatorname{dist}(A, B, C)$. From Proposition 3, since $\left.T\right|_{A}$ is $G$-continuous, we have

$$
x_{n_{k}+1}=T\left(x_{n_{k}}\right) \stackrel{G}{\longrightarrow} T\left(x^{\star}\right) .
$$

Also by the asymptotic regularly of $\left.T\right|_{A}$, we obtain

$$
\begin{aligned}
G\left(x^{\star}, T\left(x^{\star}\right), T\left(x^{\star}\right)\right) & =\lim _{k \rightarrow \infty} G\left(x_{n_{k}}, T\left(x_{n_{k}}\right), T\left(x_{n_{k}}\right)\right) \\
& =\lim _{k \rightarrow \infty} G\left(T^{n_{k}}\left(x_{0}\right), T^{n_{k}+1}\left(x_{0}\right), T^{n_{k}+1}\left(x_{0}\right)\right) \\
& =0 .
\end{aligned}
$$


This implies that $T\left(x^{\star}\right)=x^{\star}$. Since $T$ is relatively $G$-nonexpansive, we have

$$
G\left(T\left(x^{\star}\right), T\left(y^{\star}\right), T\left(z^{\star}\right)\right) \leq G\left(x^{\star}, y^{\star}, z^{\star}\right)=\operatorname{dist}(A, B, C)
$$

Therefore by the $P$-property, we have

$$
G\left(x^{\star}, T\left(x^{\star}\right), T\left(x^{\star}\right)\right)=G\left(y^{\star}, T\left(y^{\star}\right), T\left(y^{\star}\right)\right)=G\left(z^{\star}, T\left(z^{\star}\right), T\left(z^{\star}\right)\right)
$$

Hence $T\left(y^{\star}\right)=y^{\star}$ and $T\left(z^{\star}\right)=z^{\star}$.

QUESTION: In 2011, Karapinar [10] obtain some common fixed point results in partial metric spaces. Can one study the minimization problem (2.2) for two mappings in partial metric spaces?

\section{REFERENCES}

[1] M. Abbas and B. E. Rhoades, "Common fixed point results for noncommuting mappings without continuity in generalized metric spaces," Appl. Math. Comput., vol. 215, no. 1, pp. 262-269, 2009.

[2] A. Abkar and M. Gabeleh, "Global optimal solutions of noncyclic mappings in metric spaces," $J$. Optim. Theory Appl., vol. 153, no. 2, pp. 298-305, 2012.

[3] H. Aydi, E. Karapinar, and Z. Mustafa, "On common fixed points in $G$-metric spaces using (E.A) property," Comput. Math. Appl., vol. 2012, 2012.

[4] H. Aydi, E. Karapinar, and W. Shatanawi, "Tripled common fixed point results for generalized contractions in ordered generalized metric spaces," Fixed Point Theory Appl., vol. 2012, 2012.

[5] H. Aydi, E. Karapınar, and W. Shatanawi, "Tripled fixed point results in generalized metric spaces," J. Appl. Math., vol. 2012, p. 10, 2012.

[6] B. C. Dhage, "Generalised metric spaces and mappings with fixed point," Bull. Calcutta Math. Soc., vol. 84, no. 4, pp. 329-336, 1992.

[7] B. C. Dhage, "Generalized metric spaces and topological structure I." An. Ştiinţ. Univ. Al. I. Cuza Iaşi, Ser. Nouă, Mat., vol. 46, no. 1, pp. 3-24, 2000.

[8] S. Gähler, "2-metrische räume und ihre topologische struktur," Math. Nachr., vol. 26, pp. 115-148, 1963.

[9] S. Gähler, "Zur geometrie 2-metrischer räume," Rev. Roum. Math. Pures Appl., vol. 11, pp. 665667, 1966.

[10] E. Karapinar, "A note on common fixed point theorems in partial metric spaces," Math. Notes, Miskolc, vol. 12, no. 2, pp. 185-191, 2011.

[11] Z. Mustafa and B. Sims, "A new approach to generalized metric spaces," J. Nonlinear Convex Anal., vol. 7, no. 2, pp. 286-297, 2006.

[12] Z. Mustafa and B. Sims, "Fixed point theorems for contractive mappings in complete $G$-metric spaces," Fixed Point Theory Appl., vol. 2009, p. 10, 2009.

[13] V. S. Raj, "A best proximity point theorem for weakly contractive non-self-mappings," Nonlinear Anal., Theory Methods Appl., Ser. A, Theory Methods, vol. 74, no. 14, pp. 4804-4808, 2011.

[14] R. Saadati, S. M. Vaezpour, P. Vetro, and B. E. Rhoades, "Fixed point theorems in generalized partially ordered G-metric spaces," Math. Comput. Modelling, vol. 52, no. 5-6, pp. 797-801, 2010.

[15] N. Tahat, H. Aydi, E. Karapinar, and W. Shatanawi, "Common fixed points for single-valued and multi-valued maps satisfying a generalized contraction in $G$-metric spaces," Fixed Point Theory Appl., vol. 2012, 2012. 
Authors' addresses

\section{Saeed Shabani}

Department of Mathematics, Science and Research Branch, Islamic Azad University, Tehran, Iran. E-mail address: s.shabani@srbiau.ac.ir, shabani60@gmail.com

\section{Abdolrahman Razani}

Department of Mathematics, Faculty of Science, Imam Khomeini International University, P.O.Box 34149-16818, Qazvin, Iran.

E-mail address: razanilikiu.ac.ir 